\title{
Usefulness of Quantitative Bone SPECT/CT for Evaluating Treatment Response in a Patient with Mandibular Osteomyelitis
}

\author{
Kazuhiro Kitajima ${ }^{a}$ Kazuma Noguchi $^{b}$ Kuniyasu Moridera ${ }^{b}$ \\ Hiromitsu Kishimoto $^{b}$ Tatsuya Tsuchitani $^{c}$ Yoshiyuki Takahashic \\ Shungo Furudoi ${ }^{d}$ Koichiro Yamakado ${ }^{a}$ \\ ${ }^{a}$ Department of Radiology, Hyogo College of Medicine, Hyogo, Japan; bepartment of \\ Oral and Maxillofacial Surgery, Hyogo College of Medicine, Hyogo, Japan; 'Department \\ of Radiological Technology, Hyogo College of Medicine College Hospital, Hyogo, Japan; \\ dDepartment of Oral Surgery, Konan Medical Center, Hyogo, Japan
}

\section{Keywords}

Mandibular osteomyelitis · Bone scintigraphy · Quantitative single-photon emission computed tomography/computed tomography $\cdot$ Standardized uptake value

\begin{abstract}
We report here a case of mandibular osteomyelitis in a 63-year-old female in which quantitative values determined using bone SPECT/CT were useful to evaluate response to antibiotic therapy, hyperbaric oxygen therapy, and sequestomy. After finishing therapy, the chief complaints were well relieved, and posttreatment Tc-99m HMDP bone SPECT/CT examination showed decreased uptake. The maximum standardized uptake value (SUV), peak SUV, mean SUV, metabolic bone volume, and total bone uptake of the untreated lesion were 6.26, 5.16, 3.97, and $11.86 \mathrm{~mL}$ and 42.21, respectively, which were decreased to 4.65, 3.90, 2.77, and 9.67 $\mathrm{mL}$ and 26.80 , respectively, following hyperbaric oxygen therapy and antibiotic administration, and were moreover decreased to $4.28,3.67,2.75$, and $6.24 \mathrm{~mL}$ and 17.19 , respectively, after sequestomy. In comparison with pretreatment situation, those parameters were decreased by $-25.7,-24.4,-30.2,-18.5$, and $-36.5 \%$, respectively, following hyperbaric oxygen therapy and antibiotic administration, and moreover by $-31.6,-28.9,-30.7,-47.4$, and -59.3 , respectively, after sequestomy, likely reflecting treatment response. Quantitative bone SPECT/ CT may be useful to evaluate bone inflammatory activity and treatment response in a patient with mandibular osteomyelitis.
\end{abstract}




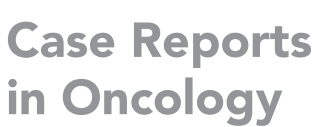

\begin{tabular}{l|l}
\hline Case Rep Oncol 2021;14:820-825 \\
\hline DOI: 10.1159/000516761 & $\begin{array}{l}\text { @ 2021 The Author(s). Published by S. Karger AG, Basel } \\
\text { www.karger.com/cro }\end{array}$ \\
\hline
\end{tabular}

Kitajima et al.: Bone SPECT/CT for Evaluating Treatment Response of Mandibular Osteomyelitis

\section{Introduction}

Mandibular osteomyelitis and septic osteonecrosis of the jaw are both infectious bone diseases that affect bone quality [1]. Histologic evaluation is nonspecific and cannot differentiate mandibular osteomyelitis from osteonecrosis of the jaw [2]. Various etiologies, such as drugs, radiation, trauma, or extraction of teeth, can cause mandibular osteomyelitis and osteonecrosis of the jaw. These conditions modifying the bone can affect the health and quality of life of patients due to infected and painful necrotic jawbone, ulcerated, painful, and swollen oral mucosa, speech disorders, and swallowing and eating difficulties. Patients with mandibular osteomyelitis and osteonecrosis of the jaw frequently require medical and dental treatments [3]. Diagnosis and therapeutic strategy are complex and challenging and need to be approached by an interdisciplinary team of oral and maxillofacial surgeons, dentists, radiooncologists, pathologists, radiologists, and nuclear medicine physicians. Furthermore, mandibular osteomyelitis and osteonecrosis of the jaw represent two of the most complicated infections of the oral and maxillofacial region, not the least because the boundary between the infected or necrotic area and healthy bone is often so ill-defined [4].

Bone scintigraphy with technetium 99m (Tc-99m) has been reported effective for detection of early-stage osteonecrosis of the jaw [5]. In addition, integration of singlephoton emission computed tomography (SPECT) with computed tomography (CT), resulting in development of hybrid SPECT/CT scanner devices, has been shown to be useful to increase diagnostic accuracy and provides accurate findings showing extent of disease in patients with medication-related osteonecrosis of the jaw and mandibular osteomyelitis $[6,7]$. Recent advances in integration of CT for attenuation correction together with a sophisticated reconstruction technique as well as positron emission tomography (PET) have enabled SPECT/CT to produce quantitative measurement findings suitable for derivation of standardized uptake value (SUV) [8]. It is anticipated that quantitative SPECT/CT will have an enormous effect on the practice of modern nuclear medicine as a clinical imaging technique [9]. In the present study, we report a case of mandibular osteomyelitis in which quantitative values determined by Tc- $99 \mathrm{~m}$ bone SPECT/CT were found useful to evaluate treatment response.

\section{Case Presentation}

A 63-year-old female who pathologically proved to be have mandibular osteomyelitis and mycotic maxillary sinusitis came to our hospital complaining of pain, swelling, and hypesthesia in the mental region. The patient had no history of bisphosphonate treatment. Tc- $99 \mathrm{~m}$ hydroxymethylene diphosphonate (Tc-99m HMDP) bone SPECT/CT revealed focal and intense uptake in the left mandibular molar region (arrow), reflecting bone inflammatory activity by mandibular osteomyelitis and extensive uptake in the right maxillary sinus due to mycetogenetic sinusitis (Fig. 1a). She underwent hyperbaric oxygen therapy (29 times) at another hospital and antibiotic administration (cefditoren pivoxil) for 3 months. The second bone SPECT/CT performed 108 days after preoperative bone SPECT/CT revealed decreased uptake in the left mandibular molar region (arrow), reflecting suppression of bone inflammatory activity (Fig. 1b). Moreover, she underwent sequestomy. The third bone SPECT/CT performed 187 days after the second bone SPECT/CT revealed mild decreased uptake in the left mandibular molar region (arrow) (Fig. 1c).

Three SPECT/CT scans using an integrated SPECT/CT system (Discovery NM/CT 670; GE Healthcare) equipped with a low-energy high-resolution collimator were performed at $3 \mathrm{~h}$ after intravenous injection of $555 \mathrm{MBq}$ of Tc-99m HMDP. SPECT/CT data were analyzed using

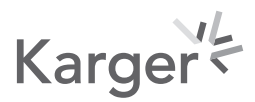



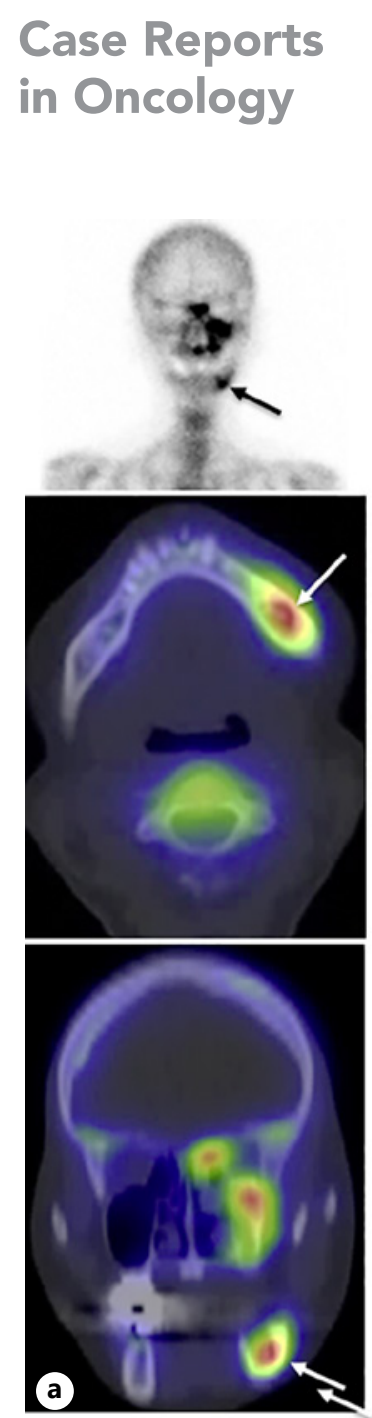

Kitajima et al.: Bone SPECT/CT for Evaluating Treatment Response of Mandibular Osteomyelitis

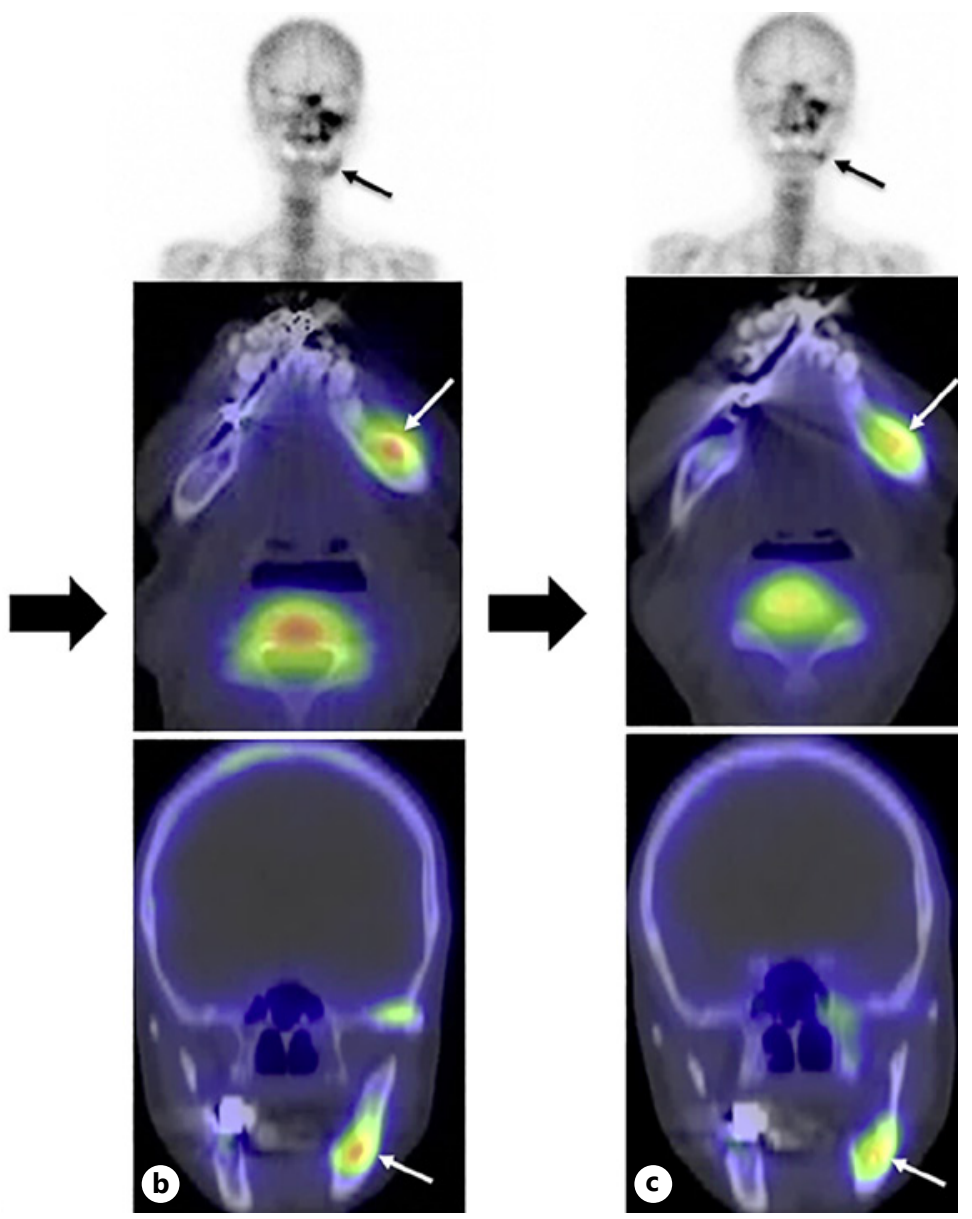

Fig. 1. Three bone SPECT/CT scans (pretreatment (a), second scan after hyperbaric oxygen therapy and antibiotic administration [cefditoren pivoxil] (b), and third scan after sequestomy (c)) show remarkable decreasing uptake in the left mandibular molar region after several therapies.

a commercially available software package (GI-BONE; Aze Co., Ltd., Tokyo, Japan), which provides statistics for various SUVs, such as max, peak, and mean SUV, as well as metabolic bone volume (MBV) and total bone uptake (TBU) [10]. SUVmax was defined as the maximum concentration in the target lesion (maximum radioactivity/voxel volume)/(injected radioactivity/body weight). SUVpeak is defined as average activity concentration within a $1 \mathrm{~cm}^{3}$ spherical VOI centered on the "hottest focus" within the tumor. SUVmean was defined as (total radioactivity/VOI volume)/(injected radioactivity/body weight). MBV (mL) was the calculated volume obtained by the accumulation of the radiopharmaceutical by placing the tumor VOI with the margin threshold set at $40 \%$ of SUVmax. TBU was then calculated as SUVmean $\times$ MBV. The SUVmax, SUVpeak, SUVmean, MBV, and TBU values of the untreated lesion were $6.26,5.16,3.97$, and $11.86 \mathrm{~mL}$ and 42.21 , respectively, which were decreased to $4.65,3.90,2.77$, and $9.67 \mathrm{~mL}$ and 26.80 , respectively, following hyperbaric oxygen therapy and antibiotic administration (cefditoren pivoxil), and were moreover decreased to 4.28 , 3.67, 2.75, and $6.24 \mathrm{~mL}$ and 17.19, respectively, after sequestomy (Fig. 2). In comparison with pretreatment situation, those parameters were decreased by $-25.7,-24.4,-30.2,-18.5$, and $-36.5 \%$, respectively, following hyperbaric oxygen therapy and antibiotic administration, and moreover by $-31.6,-28.9,-30.7,-47.4$, and $-59.3 \%$, respectively, after sequestomy. 
Kitajima et al.: Bone SPECT/CT for Evaluating Treatment Response of Mandibular Osteomyelitis

Fig. 2. The maximum SUV, peak SUV, mean SUV, MBV, and TBU of the untreated lesion were 6.26, 5.16, 3.97, and $11.86 \mathrm{~mL}$ and 42.21 , respectively, which were decreased to 4.65 , $3.90,2.77$, and $9.67 \mathrm{~mL}$ and 26.80 , respectively, following hyperbaric oxygen therapy and antibiotic administration (cefditoren pivoxil), and were moreover decreased to 4.28 , $3.67,2.75$, and $6.24 \mathrm{~mL}$ and 17.19 , respectively, after sequestomy. SUV, standardized uptake value; MBV, metabolic bone volume; TBU, total bone uptake.

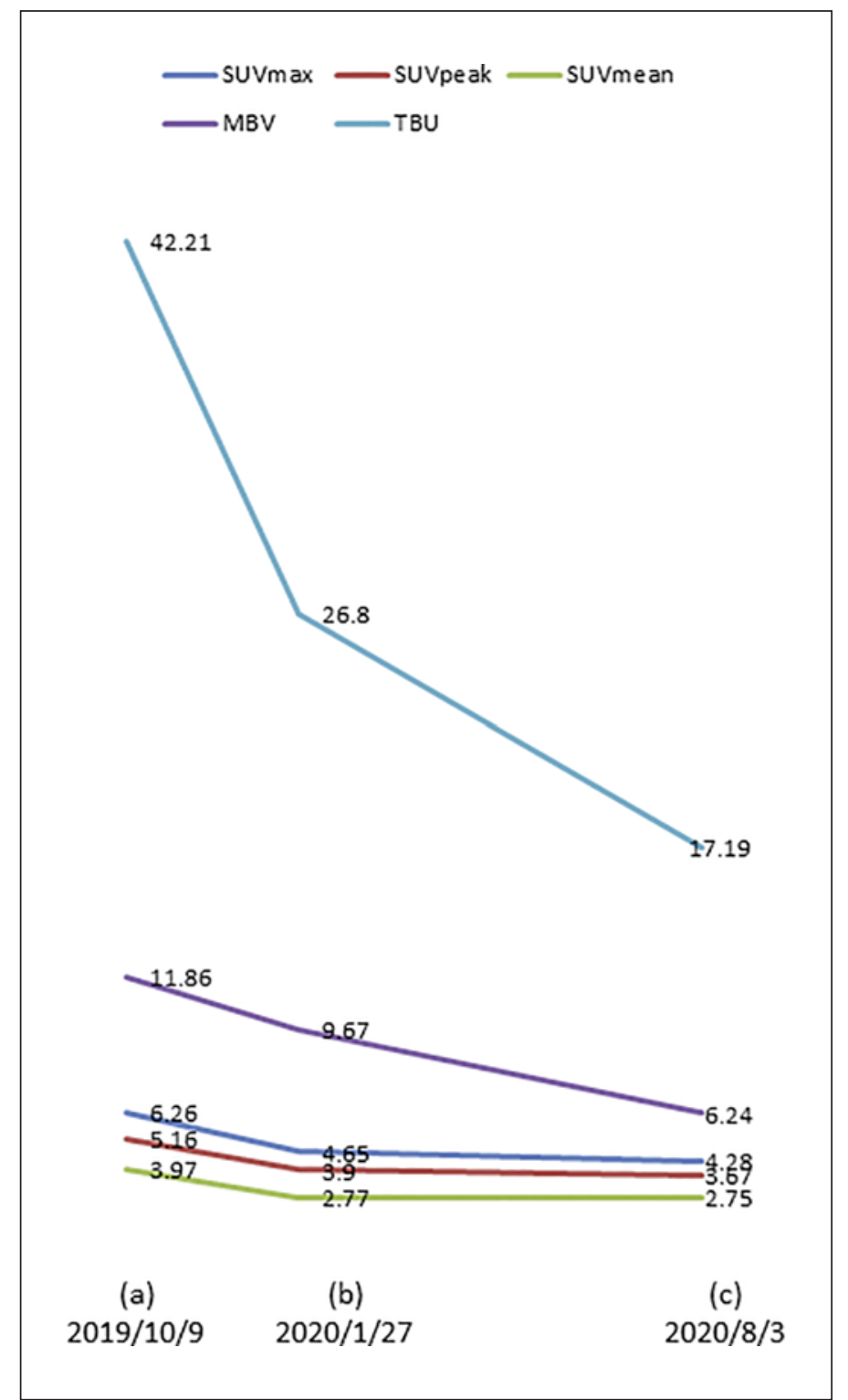

\section{Discussion}

Traditionally, gamma camera imaging has been used in nuclear medicine for 2D planar imaging, with bone scintigraphy with technetium $99 \mathrm{~m}$ phosphonates a typical example of such a technique. With development of tomographic imaging with a 3D image reconstruction algorithm, planar nuclear imaging has evolved to SPECT [11]. Because it provides in-depth information regarding radiopharmaceutical distribution, SPECT has become a major nuclear medicine modality in the fields of cardiology, neurology, oncology, and nephrology, as well as others. Quantitative SPECT/CT was subsequently developed following the successful clinical application of PET/CT findings [9].

Several studies have demonstrated clinical applications of quantitative SPECT/CT for osteonecrosis of the jaw [12-15]. Ogura et al. [12] demonstrated that SUVmax (10.16 \pm 0.96$)$, SUVpeak ( $7.95 \pm 0.88)$, SUVmean ( $5.90 \pm 0.86)$, and TBU $(94.22 \pm 57.44$ ) for chronic osteomyelitis were significantly higher than those for medication-related osteonecrosis $[5.50 \pm 2.47[p=0.020]$, 
$4.10 \pm 1.85[p=0.011], 2.74 \pm 1.07[p=0.006]$, and $29.88 \pm 15.46[p=0.034]$, respectively). The same authors [13] reported that SUVmax for medication-related osteonecrosis and rheumatoid arthritis (23.24 \pm 8.63$)$ was significantly higher than those for osteoradionecrosis $(9.05 \pm 1.39$, $p=0.005)$ and normal mandible (3.57 $\pm 0.46, p=0.0001)$. Nakai et al. [14] compared SUVmean of the 3 groups (patients treated with low-dose bisphosphonate for osteoporosis, patients treated with high-dose bisphosphonate for metastatic bone tumor, and patients with other oral disease without history of bisphosphonate treatment) and revealed that bone metabolism of the jaw and temporal bone in the bisphosphonate-treated patients was enhanced, and no suppression of bone remodeling in the jaw by bisphosphonate was observed. Hata et al. [15] evaluated pretreatment and posttreatment SUVmax and MBV in 15 patients with agent-related osteonecrosis of the jaw and demonstrated that SUVmax (from $11.5 \pm 5.9$ to $9.2 \pm 3.9, p=0.04$ ) and MBV (from $17.4 \pm 16.2 \mathrm{~cm}^{3}$ to $12.4 \pm 10.6 \mathrm{~cm}^{3}, p=0.01$ ) of all cases decreased significantly after anti-inflammatory therapy despite the short period (within 6 weeks).

\section{Conclusion}

A variety of indexes, including SUVmax, SUVpeak, SUVmean, MBV, and TBU, determined by quantitative bone SPECT/CT may be useful to evaluate bone inflammatory activity and treatment response in a patient with mandibular osteomyelitis. Additional studies with greater numbers of patients are needed to define its impact for clinical evaluation of treatment response of bony lesions.

\section{Statement of Ethics}

This report complies with the guidelines for human studies and includes evidence that the research was conducted ethically in accordance with the World Medical Association Declaration of Helsinki. The authors have no ethical conflicts to disclose. Written informed consent was obtained from the patient for publication of this case report and any accompanying images.

\section{Conflict of Interest Statement}

The authors have no conflicts of interest to declare.

\section{Funding Sources}

This work was supported by JSPS KAKENHI, Grant No. 19K08187.

\section{Author Contributions}

Concept and design: K.K. and K.Y.; acquisition of data: S.Y., K.N., K.M., T.T., Y.T., and S.F.; drafting of the manuscript: K.K.; critical revision of the manuscript for important intellectual content: K.N. and K.M.; all authors approved final version of the manuscript.

\section{Karger's}




\section{Case Reports in Oncology}

\begin{tabular}{l|l}
\hline Case Rep Oncol 2021;14:820-825 \\
\hline DOI: 10.1159/000516761 & $\begin{array}{l}\text { @ 2021 The Author(s). Published by S. Karger AG, Basel } \\
\text { www.karger.com/cro }\end{array}$ \\
\hline
\end{tabular}

Kitajima et al.: Bone SPECT/CT for Evaluating Treatment Response of Mandibular Osteomyelitis

\section{References}

1 Khalid M, Bora T, Ghaithi AA, Thukral S, Dutta J. Raman spectroscopy detects changes in bone mineral quality and collagen cross-linkage in staphylococcus infected human bone. Sci Rep. 2018;8:9417.

2 Fleisher KE, Pham S, Raad RA, Friedman KP, Ghesani M, Chan KC, et al. Does fluorodeoxyglucose positron emission tomography with computed tomography facilitate treatment of medication-related osteonecrosis of the jaw? J Oral Maxillofac Surg. 2016;74:945-58.

3 Miksad RA, Lai KC, Dodson TB, Woo SB, Treister NS, Akinyemi O, et al. Quality of life implications of bisphosphonate-associated osteonecrosis of the jaw. Oncologist. 2011;16:121-32.

4 Pautke C, Bauer F, Otto S, Tischer T, Steiner T, Weitz J, et al. Fluorescence-guided bone resection in bisphosphonate-related osteonecrosis of the jaws: first clinical results of a prospective pilot study. J Oral Maxillofac Surg. 2011;69:84-91.

5 O’Ryan FS, Khoury S, Liao W, Han MM, Hui RL, Baer D, et al. Intravenous bisphosphonate-related osteonecrosis of the jaw: bone scintigraphy as an early indicator. J Oral Maxillofac Surg. 2009;67:1363-72.

6 Miyashita H, Shiba H, Kawana H, Nakahara T. Clinical utility of three-dimensional SPECT/CT imaging as a guide for the resection of medication-related osteonecrosis of the jaw. Int J Oral Maxillofac Surg. 2015;44:1106-9.

7 Malina-Altzinger J, Klaeser B, Suter VGA, Schriber M, Vollnberg B, Schaller B. Comparative evaluation of SPECT/CT and CBCT in patients with mandibular osteomyelitis and osteonecrosis. Clin Oral Investig. 2019; 23:4213-22.

8 Zeintl J, Vija AH, Yahil A, Hornegger J, Kuwert T. Quantitative accuracy of clinical 99mTc SPECT/CT using ordered-subset expectation maximization with 3-dimensional resolution recovery, attenuation, and scatter correction. J Nucl Med. 2010;51:921-8.

9 Bailey DL, Willowson KP. An evidence-based review of quantitative SPECT imaging and potential clinical applications. J Nucl Med. 2013;54:83-9.

10 Kitajima K, Futani H, Fujiwara M, Minakawa G, Osugi Y, Tsuchitani T, et al. Usefulness of quantitative bone single photon emission computed tomography/computed tomography for evaluating response to neoadjuvant chemotherapy in a patient with periosteal osteosarcoma. Cureus. 2018;10(11):e3655.

11 Madsen MT. Recent advances in SPECT imaging. J Nucl Med. 2007;48:661-73.

12 Ogura I, Kobayashi E, Nakahara K, Igarashi K, Haga-Tsujimura M, Toshima H. Quantitative SPECT/CT imaging for medication-related osteonecrosis of the jaw: a preliminary study using volume-based parameters, comparison with chronic osteomyelitis. Ann Nucl Med. 2019;33:776-82.

13 Ogura I, Sasaki Y, Sue M, Oda T, Kameta A, Hayama K. Tc-99m hydroxymethylene diphosphonate SPECT/CT for the evaluation of osteonecrosis of the jaw: preliminary study on diagnostic ability of maximum standardised uptake value. Clin Radiol. 2020;75:46-50.

14 Nakai F, Ohbayashi Y, Nakai Y, Iwasaki A, Miyake M. Bone metabolism of the jaw in response to bisphosphonate: a quantitative analysis of bone scintigraphy images. Odontology. 2020;108:653-60.

15 Hata H, Kitao T, Sato J, Asaka T, Ohga N, Imamachi K, et al. Monitoring indices of bone inflammatory activity of the jaw using SPECT bone scintigraphy: a study of ARONJ patients. Sci Rep. 2020;10:11385. 